PREPARED FOR THE U.S. DEPARTMENT OF ENERGY, UNDER CONTRACT DE-AC02-76CH03073

PPPL-3935

PPPL-3935

UC-70

\author{
Omniclassical Diffusion \\ in Low Aspect Ratio Tokamaks
}

by

H.E. Mynick, R.B. White, and D.A. Gates

March 2004

$\left.\stackrel{M}{M}\right|_{\substack{\text { PRInCETON PLASIMA } \\ \text { PHYSICS LABORATORY }}} ^{D}$

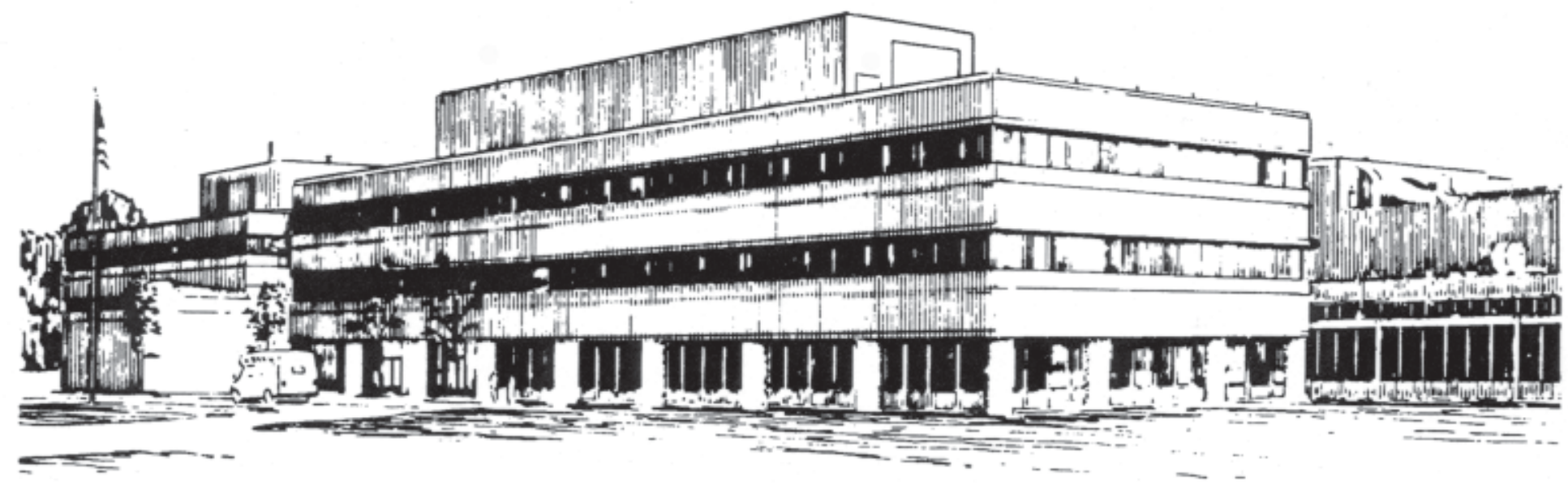

PRINCETON PLASMA PHYSICS LABORATORY PRINCETON UNIVERSITY, PRINCETON, NEW JERSEY 


\section{PPPL Reports Disclaimer}

This report was prepared as an account of work sponsored by an agency of the United States Government. Neither the United States Government nor any agency thereof, nor any of their employees, makes any warranty, express or implied, or assumes any legal liability or responsibility for the accuracy, completeness, or usefulness of any information, apparatus, product, or process disclosed, or represents that its use would not infringe privately owned rights. Reference herein to any specific commercial product, process, or service by trade name, trademark, manufacturer, or otherwise, does not necessarily constitute or imply its endorsement, recommendation, or favoring by the United States Government or any agency thereof. The views and opinions of authors expressed herein do not necessarily state or reflect those of the United States Government or any agency thereof.

\section{Availability}

This report is posted on the U.S. Department of Energy's Princeton Plasma Physics Laboratory Publications and Reports web site in Fiscal Year 2004. The home page for PPPL Reports and Publications is: http://www.pppl.gov/pub_report/

DOE and DOE Contractors can obtain copies of this report from:

U.S. Department of Energy

Office of Scientific and Technical Information

DOE Technical Information Services (DTIS)

P.O. Box 62

Oak Ridge, TN 37831

Telephone: (865) 576-8401

Fax: (865) 576-5728

Email: reports@adonis.osti.gov

This report is available to the general public from:

National Technical Information Service

U.S. Department of Commerce

5285 Port Royal Road

Springfield, VA 22161

Telephone: $1-800-553-6847$ or

(703) $605-6000$

Fax: (703) 321-8547

Internet: http://www.ntis.gov/ordering.htm 


\title{
Omniclassical Diffusion in Low Aspect Ratio Tokamaks
}

\author{
H.E. Mynick, R.B. White, \& D.A. Gates \\ Plasma Physics Laboratory, Princeton University \\ P.O. Box 451 \\ Princeton, New Jersey 08543-0451, U.S.A.
}

\begin{abstract}
Recently reported numerical results for axisymmetric devices with low aspect ratio $A$ found radial transport enhanced over the expected neoclassical value by a factor of 2 to 3 . In this paper, we provide an explanation for this enhancement. Transport theory in toroidal devices usually assumes large $A$, and that the ratio $B_{p} / B_{t}$ of the poloidal to the toroidal magnetic field is small. These assumptions result in transport which in the low collision limit is dominated by banana orbits, giving the largest collisionless excursion of a particle from an initial flux surface. However in a small aspect ratio device one may have $B_{p} / B_{t} \sim 1$, and the gyroradius may be larger than the banana excursion. Here, we develop an approximate analytic transport theory valid for devices with arbitrary $A$. For low $A$, we find that the enhanced transport, referred to as omniclassical, is a combination of neoclassical and properly generalized classical effects, which become dominant in the low- $A, B_{p} / B_{t} \sim 1$ regime. Good agreement of the analytic theory with numerical simulations is obtained.
\end{abstract}

PACS numbers: 52.25.Fi 52.65.Cc 52.55.Fa 
A recent numerical study ${ }^{1}$ of collisional transport in tokamaks with low aspect ratio ("spherical tori") found that the transport rate predicted by a Lorentz (i.e., full-orbit) code GYROXY2 is substantially larger than the rate computed using guiding-center (GC) codes such as ORBIT. ${ }^{3}$ The drift-kinetic equations used by GC codes are also the basis of standard neoclassical theory, ${ }^{4,5}$ so the disparity between the GC and Lorentz results indicates that something is missing in neoclassical theory, related to the effects of finite gyroradius for devices with low aspect ratio $A \equiv R / a$ ( $R$ and $a$ are the device major and minor radii), and larger ratios $B_{p} / B_{t}$ of the poloidal to toroidal magnetic field than those found in larger- $A$ tokamaks. The total transport including these new effects was termed "omniclassical transport."

In this work, we provide an explanation for the omniclassical enhancement over the neoclassical rates from the ORBIT code. The explanation is a generalization of the classical transport one expects in a 1-dimensional (1D) or large- $A$ (weakly 2D) system, where the diffusion coefficient $D_{c l} \simeq \nu \rho_{g}^{2}$ is small compared with the neoclassical coefficient $D_{n c} \simeq \nu\left(q \rho_{g}\right)^{2} / \epsilon^{3 / 2}$ (with $\nu$ the collision frequency, $\rho_{g}$ the particle gyroradius, and $\epsilon \equiv A^{-1}$ ). While some form of classical transport is one natural candidate to explain the numerical results in Ref. 1, how this mechanism generalizes to the strongly 2D case, and whether this in fact accounts for the observed disparity, need to be determined. Here, we present an approximate analytic calculation of the radial transport, from which emerges the appropriately generalized expression for the classical transport in this strongly 2D context, and we provide the demonstration that this expression accounts for the numerical findings.

In the GC code, a collision operator $C=C_{\|}$is used which scatters only in pitch $\lambda \equiv v_{\|} / v_{\perp} \equiv v \cos \theta_{v}$, where $v$ is the particle speed, and $v_{\|}$and $v_{\perp}$ are its components parallel and perpendicular to the magnetic field $\mathbf{B}$. This permits the particle "banana center" (bounce-averaged flux surface label) $\bar{x}$ to wander diffusively. In GYROXY, as well as in the analytic calculation, $C$ scatters not only 
in $\lambda$, but also in gyrophase $\phi_{v}, C=C_{\|}+C_{\perp}$, providing a second statistically independent process by which $\bar{x}$ can wander, enhancing the overall transport. As in the 1D case, the analytic theory finds that the classical transport has two contributions, one from scattering in $\lambda$, and a dominant contribution from scattering in $\phi_{v}$. When the collision operator in GYROXY is constrained to scatter only in $\lambda$, we find that the transport rate drops toward that from ORBIT by an amount consistent with the theory.

The method used to compute $D$ with ORBIT and GYROXY is as described in Ref. 1. A monoenergetic distribution of particles is loaded on a surface $\psi_{0}(\psi$ is the poloidal flux $/ 2 \pi$ ) uniformly in poloidal angle $\theta$ and pitch variable $\lambda$. Integrating the particle orbits, $D$ is computed from the Fokker-Planck expression $D=(1 / 2) d / d t\left\langle(\delta \psi)^{2}\right\rangle(t)$. In Fig. 1 we plot $\left\langle(\delta \psi)^{2}\right\rangle$ (arbitrary units) versus time from GYROXY and ORBIT, for a high beta equilibrium in the National Spherical Torus Experiment ${ }^{6}$ (NSTX) spherical torus with a $100 \mathrm{eV}$ monoenergetic distribution launched on a surface $\psi$ with maximum major radius $X(\psi)=140 \mathrm{~cm}$. The collision frequency used is $10^{-4} \omega_{0}$, with $\omega_{0}$ the cyclotron frequency, which is well within the banana regime. The top curve (a) is from GYROXY with full collision operator $C=C_{\|}+C_{\perp}$, the bottom curve (c) is from ORBIT, which has $C_{\|}$only, and the middle curve (b) is from GYROXY with $C_{\|}$only. One notes that the slope of this curve has dropped most of the way from that of full- $C$ GYROXY curve to the ORBIT curve, as indicated above. The rapid displacement from the flux surface occuring initially reflects the mean square banana width in the case of ORBIT, and the much larger banana plus gyro width for the upper two curves. In Fig. 2 we plot $D / D_{n c}$ versus $X(\psi)$, where $D_{n c}$ is the GC result from ORBIT. The top curve (a) gives results using the full $C$, and the bottom curve (b) with $C$ constrained to $C_{\|}$only. Doing so removes about $3 / 4$ of the disparity between the GYROXY and ORBIT results.

To gain an analytic understanding of these results, we consider the diffusion of particles in the space of the three constants of motion $\mathbf{J}$, and in particular, 
diffusion in the banana-center flux surface $\bar{x}$. (A typical choice for $\mathbf{J}$, employed in the "action-angle formalism", 7 are the magnetic moment $J_{g}$, the bounce action $J_{b}$, and the angular momentum $p_{\zeta}$. For our limited purposes here, we shall only need to make use of a small part of the machinery of this formalism.) One may define $\bar{x}$ using the conservation of $p_{\zeta} \equiv \mathbf{e}_{\zeta} \cdot \mathbf{p} \equiv \frac{e}{c} A_{\zeta}+M v_{\zeta}$ for collisionless orbits. Here, $\mathbf{e}_{\zeta}=R \hat{\zeta}$ is the contravariant basis vector for toroidal azimuth $\zeta$. We use a flux coordinate system $(x, \theta, \zeta)$, with $x$ the flux-surface label. The vector potential is given by $\mathbf{A}=\Phi \nabla \theta-\psi \nabla \zeta$, with $A_{\theta}(x)=\Phi$ the toroidal flux $/ 2 \pi$, and $A_{\zeta}(x)=-\psi$. Thus, $p_{\zeta}=-\frac{e}{c} \psi+M v_{\zeta}$, and for this reason, expressions are somewhat simpler if one adopts the choice $x \rightarrow \psi$, which we now do. Since $p_{\zeta}$ is a constant of the motion, it equals its orbit-average $\overline{p_{\zeta}}=\frac{e}{c} \bar{\psi}+M \bar{v}_{\zeta}$, and thus

$$
\bar{\psi}=-\frac{c}{e}\left(p_{\zeta}-M \bar{v}_{\zeta}\right)=\psi-\frac{c}{e} M\left(v_{\zeta}-\overline{v_{\zeta}}\right) .
$$

After a little geometry, one can write $v_{\zeta}$ in terms of the more conventional components $v_{\|}, v_{\perp}$ as

$$
v_{\zeta}=R\left(b_{t} v_{\|}+b_{p} v_{\perp} \cos \phi_{v}\right)=R v\left(b_{t} \lambda+b_{p} \sqrt{1-\lambda^{2}} \cos \phi_{v}\right) \equiv v_{\zeta \|}+v_{\zeta \perp},
$$

where $b_{t, p} \equiv B_{t, p} / B$, the ratio of the toroidal or poloidal to the total magnetic field.

For trapped particles (trapping-state index $\tau=0$ ), one has $\overline{v_{\zeta}}=0$. For passing particles $(\tau=1), v_{\bar{\zeta} \perp}$ again vanishes under the gyro-average, while $\overline{v_{\zeta}} \simeq v_{\zeta \|}$, an approximation improving for more deeply-passing particles. Thus, in Eq.(1), one has

$$
\left(v_{\zeta}-\bar{v}_{\zeta}\right) \simeq(1-\tau) v_{\zeta \|}+v_{\zeta \perp}
$$

As in the GYROXY simulations, we adopt a collision operator which scatters in $\lambda$ and $\phi_{v}$, but not in energy:

$C \equiv C_{\|}+C_{\perp} \equiv\left(\nu v^{2} / 2\right) \nabla_{\mathbf{v}} \cdot(\mathbf{I}-\hat{\mathbf{v}} \hat{\mathbf{v}}) \cdot \nabla_{\mathbf{v}}=\nu / 2\left[\partial_{\lambda}\left(1-\lambda^{2}\right) \partial_{\lambda}+\left(1-\lambda^{2}\right)^{-1 / 2} \partial_{\phi_{v}}^{2}\right]$. 
$C_{\|}$is the usual Lorentz collison operator used in the drift-kinetic framework. Both $C_{\|}, C_{\perp}$ are self-adjoint in $\left(\lambda, \phi_{v}\right)$ space: for any functions $F, G$, $\int d^{2} \mathbf{v} F C_{\|, \perp} G=\int d^{2} \mathbf{v} G C_{\|, \perp} F$.

We compute the radial flux of banana centers due to the action of $C$ : $d_{t}\langle\bar{\psi}\rangle \equiv\langle C \bar{\psi}\rangle$, where $d_{t}$ is the collisionally-induced time derivative, and for any function $F(z),\langle F\rangle \equiv V^{-1} \int_{V} d^{6} z f F$ is a phase-space integral $\left(d^{6} z=d^{3} \mathbf{x} d^{3} \mathbf{p}\right)$ of $F$ weighted by distribution function $f$, over a thin toroidal shell of volume $V$ centered at flux surface $\psi$. From Eq.(4), one has $d_{t}=d_{t}^{\|}+d_{t}^{\perp}$, yielding contributions to the flux induced by $C_{\|}$and $C_{\perp}$, respectively. From Eqs.(1)-(4), one thus has

$$
d_{t}\langle\bar{\psi}\rangle=\left(-\frac{c}{e} M\right) d_{t}\left\langle\left(v_{\zeta}-\bar{v}_{\zeta}\right)\right\rangle=\left(-\frac{c}{e} M\right)\left\langle\left(C_{\|}+C_{\perp}\right)\left[(1-\tau) v_{\zeta \|}+v_{\zeta \perp}\right]\right\rangle,
$$

which one sees gives four terms, of which the term $\left\langle C_{\perp}(1-\tau) v_{\zeta \|}\right\rangle$ vanishes. The first term is the neoclassical flux, $\Gamma_{n c} \equiv\left(-\frac{c}{e} M\right)\left\langle\left(C_{\|}(1-\tau) v_{\zeta \|}\right\rangle\right.$, while the remaining two yield the generalized classical flux, $\left.\Gamma_{c l} \equiv\left(-\frac{c}{e} M\right)\left\langle\left(C_{\|}+C_{\perp}\right) v_{\zeta \perp}\right]\right\rangle$.

To complete the calculation, we need an expression for the particle distribution $f$. Any function $f=f_{0}(\mathbf{J})$ of $\mathbf{J}$ satisfies the Vlasov equation, so for low $\nu$, a good collisionless approximation is the local Maxwellian form $f_{0}(\bar{\psi}, E)=$ $n_{0} /[2 \pi T M]^{3 / 2} \exp (-E / T)$, with $n_{0}$ and $T$ functions of $\bar{\psi}$, and $E \equiv M v^{2} / 2$ the particle energy. Using $\bar{\psi}=\psi-\delta \psi$, one has $f_{0}(\bar{\psi}) \simeq f_{0}(\psi)-\delta \psi \partial_{\psi} f_{0}$, where $\partial_{\psi} f_{0}=-\kappa f_{0}$, with $\kappa=\kappa_{n}+\kappa_{T}\left(v^{2} / v_{T}^{2}-3\right) / 2, \kappa_{n} \equiv-\partial_{\psi} \ln n_{0}$ and $\kappa_{T} \equiv-\partial_{\psi} \ln T$. From Eqs.(1) and (3), $\delta \psi=\left(\frac{c}{e} M\right)\left((1-\tau) v_{\zeta \|}+v_{\zeta \perp}\right) \equiv \delta \psi_{b}+\delta \psi_{g}$, with $\delta \psi_{g}$ the gyro-orbit radial excursion, and $\delta \psi_{b}$ the (bounce-related) radial drift excursion. Using these in (5), one finds

$$
\begin{aligned}
d_{t}\langle\bar{\psi}\rangle= & -V^{-1} \int_{V} d^{6} z \partial_{\psi} f_{0} \frac{\nu}{2}\left(\frac{c}{e} M R v\right)^{2} \times \\
& \left\{(1-\tau) b_{t}^{2}\left(1-\lambda^{2}\right)+b_{p}^{2}\left[\left(1-2 \lambda^{2}\right)+\sqrt{1-\lambda^{2}}\right] \cos ^{2} \phi_{v}\right\}
\end{aligned}
$$

The integrations over $\lambda$ and $\phi_{v}$ here are elementary, and those over $\theta$ and $v$ may be done for specific models. Setting $\kappa_{T}=0$ to extract only the diagonal term $D^{\psi}$ 
of the transport matrix, we find from Eq.(6)

$$
d_{t}\langle\bar{\psi}\rangle=-\partial_{\psi} n_{0}\left[\bar{D}_{n c}^{\psi}(\psi)+\bar{D}_{c l}^{\psi}(\psi)\right]=\kappa_{n} V^{-1} \int_{V} d^{6} z f_{0}\left[D_{n c}^{\psi}(v, \mathbf{x})+D_{c l}^{\psi}(v, \mathbf{x})\right],
$$

where $D_{n c}^{\psi}(v, \mathbf{x})=\frac{1}{2} \nu \rho_{g}^{2}\left(B_{t} R\right)^{2}\left(1-B / B_{\pi}\right)^{1 / 2}$, and

$D_{c l}^{\psi}(v, \mathbf{x})=\frac{1}{4}\left(\frac{1}{3}+\frac{\pi}{2}\right) \nu \rho_{g}^{2}\left(B_{p} R\right)^{2}$ are the neoclassical and generalized classical diffusion coefficients, which must be integrated over $\theta$ and $v$ to obtain the averaged coefficients. Here, $B_{\pi} \equiv B(\psi, \theta=\pi)$ is the maximum value of $B$ on surface $\psi$. The factor $\frac{1}{3}$ in $D_{c l}^{\psi}$ is the contribution from the term $C_{\|} v_{\zeta \perp}$ in (5), while the dominant factor $\frac{\pi}{2}$ is from the term $C_{\perp} v_{\zeta \perp}$. Thus, removing $C_{\perp}$ from the GYROXY simulation should remove most of the enhancement of $D$ over $D_{n c}$, as seen in Fig. 2. The dominant contribution from this approximate theory is about $\frac{\pi}{2} /\left(\frac{1}{3}+\frac{\pi}{2}\right) \simeq 82 \%$ of $D_{c l}$, while the factor in Fig. 2 is about $70 \%$. Note that ratios of these quantities are independent of particle energy, being due to equilibrium field geometry, and thus these results apply to heat transport as well as particle transport.

We put these diffusion coefficients in a more familiar form by transforming from $\psi$ to a flux function $r(\psi)$ having units of length, which approximates an average minor radius. One has $d_{t}\langle\bar{r}\rangle=-\partial_{r} n_{0} \bar{D}^{r}$, with $\bar{D}^{r}=\left(\partial_{\psi} r\right)^{2} \bar{D}^{\psi}$, and similarly for $D^{r}$. Taking $r \equiv \sqrt{2 \Phi / B_{0}}$, with $B_{0}$ the toroidal field strength on the magnetic axis, one has $\left(\partial_{\psi} r\right)=\left(q / B_{0} r\right)$, and thus

$D_{n c}^{r}(v, \mathbf{x})=\frac{1}{2} \nu\left(\rho_{g} q\right)^{2}\left(B_{t} R / B_{0} r\right)^{2}\left(1-B / B_{\pi}\right)^{1 / 2}$, and $D_{c l}^{r}(v, \mathbf{x})=\frac{1}{4}\left(\frac{1}{3}+\frac{\pi}{2}\right) \nu \rho_{g}^{2}\left(q B_{p} R / B_{0} r\right)^{2}$.

The $\theta$-dependences in these expressions lie in $\rho_{g},\left(1-B / B_{\pi}\right)$, and $B_{p} R$. For a small- $\epsilon$ device, $\rho_{g} \simeq$ const, $\oint \frac{d \theta}{2 \pi}\left(1-B / B_{\pi}\right)^{1 / 2} \simeq \frac{2}{\pi} \sqrt{2 \epsilon},\left(B_{t} R / B_{0} r\right)^{2} \simeq 1 / \epsilon^{2}$, and $\left(q B_{p} R / B_{0} r\right)^{2} \simeq 1$, resulting in the familiar dependences $D_{n c}^{r}(v, \mathbf{x}) \simeq \sigma_{n c} \nu\left(\rho_{g} q\right)^{2} / \epsilon^{3 / 2}$ and $D_{c l}^{r}(v, \mathbf{x}) \simeq \sigma_{c l} \nu \rho_{g}^{2}$, with numerical coefficients $\sigma_{n c}=\frac{\sqrt{2}}{\pi}$, and $\sigma_{c l}=\frac{1}{4}\left(\frac{1}{3}+\frac{\pi}{2}\right)$.

In Fig. 3 we average these expressions using the NSTX geometry to compute the analytic counterparts of the numerical results in Fig. 2. One notes the approx- 
imate agreement of the numerical and analytic results.

Summarizing, we have provided an explanation for the omniclassical enhancement of the total transport over neoclassical rates observed in Ref. 1, and developed an approximate transport theory which predicts both the enhancement over neoclassical from the full-orbit GYROXY code with full collision operator, and the residual finite-gyroradius enhancement in GYROXY present when its collision operator is constrained to scatter in pitch only. The enhancement comes from a generalization to strongly $2 \mathrm{D}$ geometries of classical transport, which for low- $A$ configurations like NSTX can dominate over the neoclassical contribution.

\section{Acknowledgment}

This work supported by U.S.Department of Energy Contract No.DE-AC0276-CHO3073. 


\section{References}

${ }^{1}$ D.A. Gates, R.B. White (submitted for publication, Jan. 2004).

${ }^{2}$ L. Chen, Z. Lin, R.B. White, Phys. Plasmas8 4713 (2001).

${ }^{3}$ R.B. White, M.S. Chance,Phys. Fluids 27, 2455 (1984).

${ }^{4}$ F.L. Hinton, R.D. Hazeltine, Rev. Mod. Phys 48, Part 1, 239 (1976).

${ }^{5}$ C.S. Chang, F.L. Hinton, Phys. Fluids 25, 1493 (1982).

${ }^{6}$ M. Ono et al, Nucl Fusion 40, 557 (2000).

${ }^{7}$ A. N. Kaufman, Phys. Fluids 15, 1063 (1972). 


\section{Figures}

FIG. 1. Plots of $\left\langle(\delta \psi)^{2}\right\rangle$ versus time from the full-orbit code GYROXY and guidingcenter code ORBIT, from whose slopes (straight lines) the diffusion coefficients are computed. See text for details.

FIG. 2. The ratio $D / D_{n c}$ of the diffusion coefficient from GYROXY to that from ORBIT, versus radial variable $X$. See text for details.

FIG. 3. The same ratio $D / D_{n c}$ as in Fig. 2 from the analytic expressions derived in the text. 


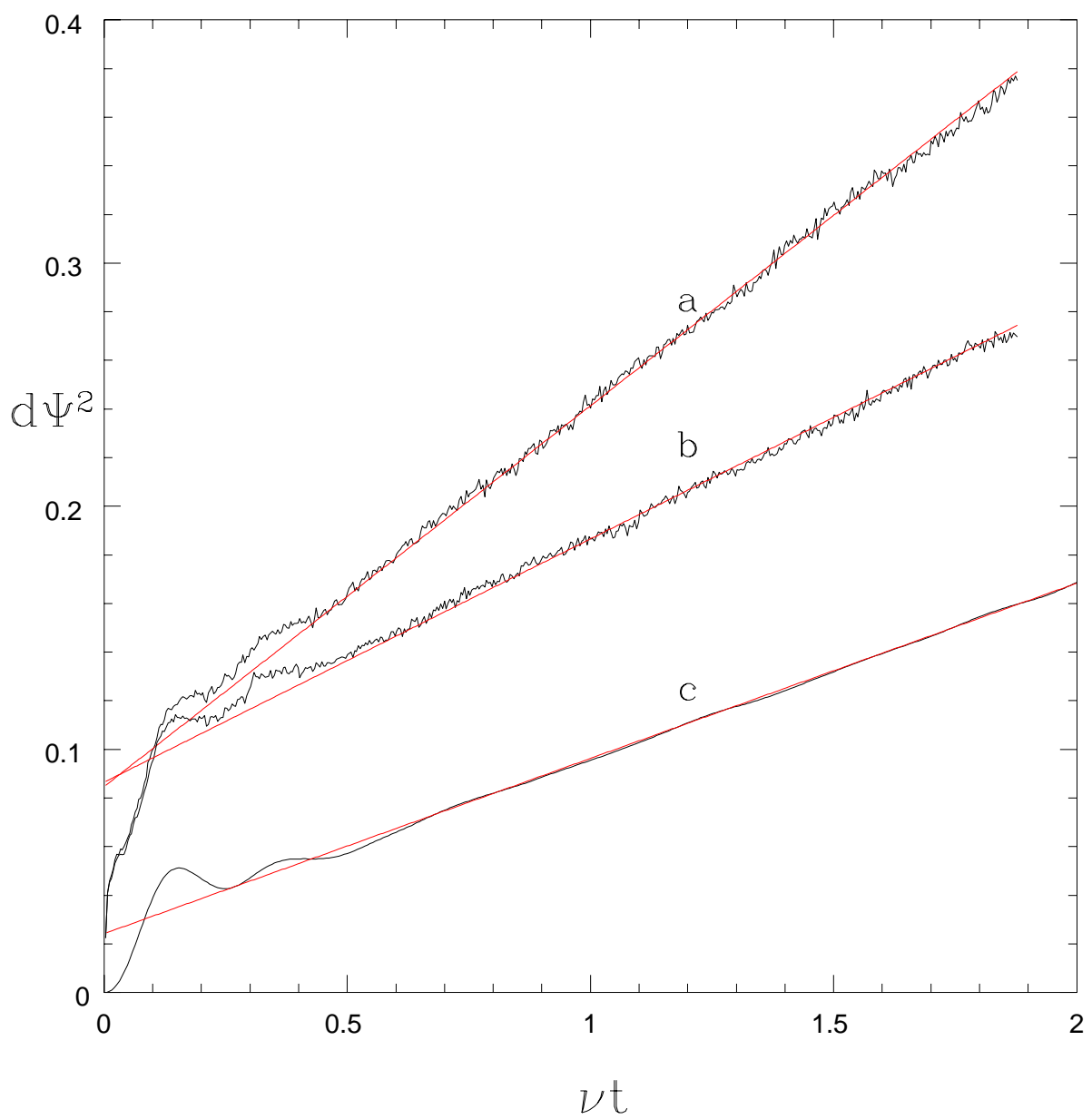

Figure 1: 


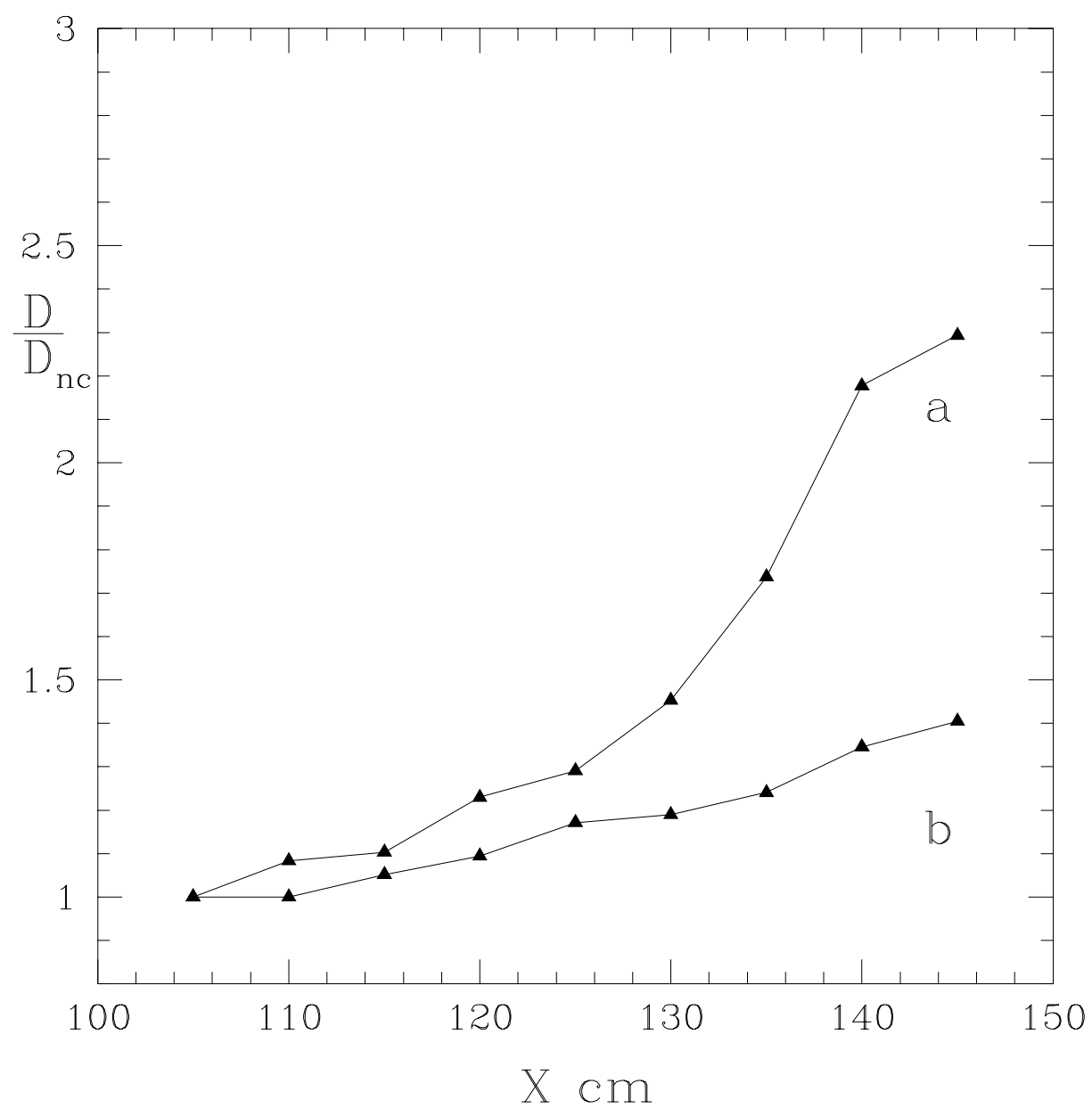

Figure 2: 


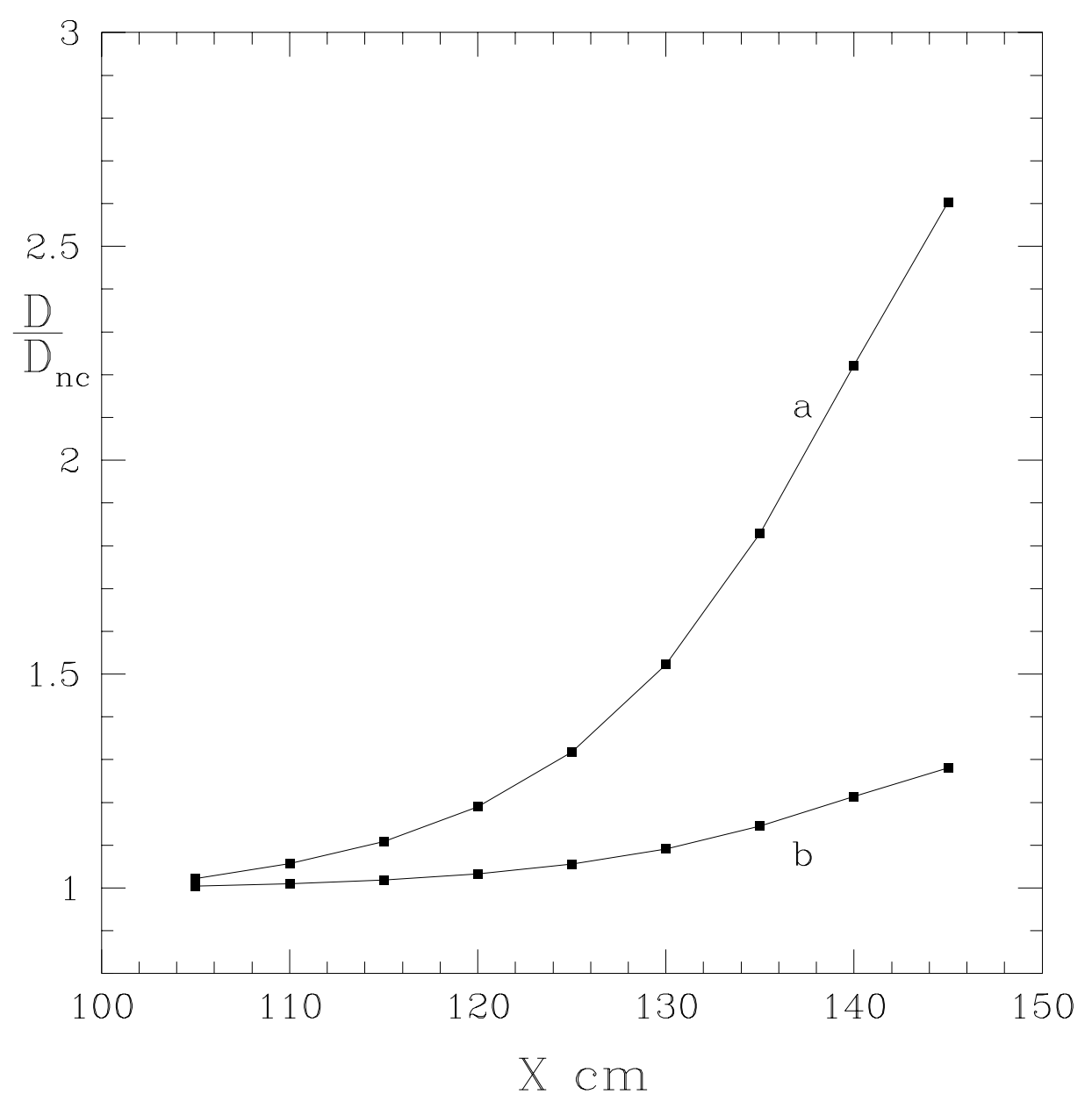

Figure 3: 


\section{External Distribution}

Plasma Research Laboratory, Australian National University, Australia

Professor I.R. Jones, Flinders University, Australia

Professor João Canalle, Instituto de Fisica DEQ/IF - UERJ, Brazil

Mr. Gerson O. Ludwig, Instituto Nacional de Pesquisas, Brazil

Dr. P.H. Sakanaka, Instituto Fisica, Brazil

The Librarian, Culham Laboratory, England

Mrs. S.A. Hutchinson, JET Library, England

Professor M.N. Bussac, Ecole Polytechnique, France

Librarian, Max-Planck-Institut für Plasmaphysik, Germany

Jolan Moldvai, Reports Library, Hungarian Academy of Sciences, Central Research Institute for Physics, Hungary

Dr. P. Kaw, Institute for Plasma Research, India

Ms. P.J. Pathak, Librarian, Institute for Plasma Research, India

Ms. Clelia De Palo, Associazione EURATOM-ENEA, Italy

Dr. G. Grosso, Instituto di Fisica del Plasma, Italy

Librarian, Naka Fusion Research Establishment, JAERI, Japan

Library, Laboratory for Complex Energy Processes, Institute for Advanced Study, Kyoto University, Japan

Research Information Center, National Institute for Fusion Science, Japan

Dr. O. Mitarai, Kyushu Tokai University, Japan

Dr. Jiangang Li, Institute of Plasma Physics, Chinese Academy of Sciences, People's Republic of China

Professor Yuping Huo, School of Physical Science and Technology, People's Republic of China

Library, Academia Sinica, Institute of Plasma Physics, People's Republic of China

Librarian, Institute of Physics, Chinese Academy of Sciences, People's Republic of China

Dr. S. Mirnov, TRINITI, Troitsk, Russian Federation, Russia

Dr. V.S. Strelkov, Kurchatov Institute, Russian Federation, Russia

Professor Peter Lukac, Katedra Fyziky Plazmy MFF UK, Mlynska dolina F-2, Komenskeho Univerzita, SK-842 15 Bratislava, Slovakia

Dr. G.S. Lee, Korea Basic Science Institute, South Korea

Institute for Plasma Research, University of Maryland, USA

Librarian, Fusion Energy Division, Oak Ridge National Laboratory, USA

Librarian, Institute of Fusion Studies, University of Texas, USA

Librarian, Magnetic Fusion Program, Lawrence Livermore National Laboratory, USA

Library, General Atomics, USA

Plasma Physics Group, Fusion Energy Research Program, University of California at San Diego, USA

Plasma Physics Library, Columbia University, USA

Alkesh Punjabi, Center for Fusion Research and Training, Hampton University, USA

Dr. W.M. Stacey, Fusion Research Center, Georgia Institute of Technology, USA

Dr. John Willis, U.S. Department of Energy, Office of Fusion Energy Sciences, USA

Mr. Paul H. Wright, Indianapolis, Indiana, USA 
The Princeton Plasma Physics Laboratory is operated by Princeton University under contract with the U.S. Department of Energy.

\author{
Information Services \\ Princeton Plasma Physics Laboratory \\ P.O. Box 451 \\ Princeton, NJ 08543
}

Phone: 609-243-2750

Fax: 609-243-2751

e-mail: pppl_info@pppl.gov

Internet Address: http://www.pppl.gov 